\title{
IDENTIFYING MINIMAL AND DOMINANT SOLUTIONS FOR KUMMER RECURSIONS
}

\author{
ALFREDO DEAÑO, JAVIER SEGURA, AND NICO M. TEMME
}

\begin{abstract}
We identify minimal and dominant solutions of three-term recurrence relations for the confluent hypergeometric functions ${ }_{1} F_{1}\left(a+\epsilon_{1} n ; c+\right.$ $\left.\epsilon_{2} n ; z\right)$ and $U\left(a+\epsilon_{1} n, c+\epsilon_{2} n, z\right)$, where $\epsilon_{i}=0, \pm 1$ (not both equal to 0 ). The results are obtained by applying Perron's theorem, together with uniform asymptotic estimates derived by T. M. Dunster for Whittaker functions with large parameter values. The approximations are valid for complex values of $a$, $c$ and $z$, with $|\mathrm{ph} z|<\pi$.
\end{abstract}

\section{INTRODUCTION}

Hypergeometric functions of Gauss and Kummer type satisfy three-term recurrence relations that connect three functions of the same family whose parameters differ by integer numbers. These identities are an important part of the theory of hypergeometric functions, and play an essential role in some special subfamilies, like the classical orthogonal polynomials.

From a numerical and computational perspective, three-term recurrence relations are a valuable tool, since in principle they allow us to compute a hypergeometric function with given values of the parameters by using two starting values and applying an appropriate recursion. However, as is discussed in detail in [3] and [4, Ch. 4], great care is needed in order to use these recursions numerically in a stable way.

We recall that given a three-term recurrence relation of the form

$$
y_{n+1}(z)+b_{n} y_{n}(z)+a_{n} y_{n-1}(z)=0,
$$

where $n$ is an integer parameter, a solution $f_{n}(z)$ is said to be minimal (or recessive) when $n \rightarrow+\infty$ if

$$
\lim _{n \rightarrow+\infty} \frac{f_{n}(z)}{g_{n}(z)}=0,
$$

for any other solution $g_{n}(z)$ of (1.1) that is linearly independent of $f_{n}(z)$. The solution $g_{n}(z)$ is said to be dominant. In some cases the recursion (1.1) can also be used for $n \rightarrow-\infty$, and a different pair of dominant and minimal solutions may arise.

Received by the editor August 30, 2007.

2000 Mathematics Subject Classification. Primary 33C15, 39A11, 41A60, 65D20.

Key words and phrases. Kummer functions, Whittaker functions, confluent hypergeometric functions, recurrence relations, difference equations, stability of recurrence relations, numerical evaluation of special functions, asymptotic analysis.

(C)2008 American Mathematical Society Reverts to public domain 28 years from publication 
Whenever a three term recursion admits a minimal solution, a numerically satisfactory pair [4] is a pair of linearly independent solutions that includes the minimal one. The importance of such a pair is due to the fact that it can be used to compute numerically any other solution of the recursion. On the other hand, as we will see for some of the Kummer recursions discussed in this paper, it may happen that we can find two linearly independent dominant solutions, that can be computed rather easily. Such a pair cannot be used to compute the minimal solution, because of numerical cancellation, and they do not constitute a numerically satisfactory pair.

It is not difficult to verify that the minimal solution of a three-term recursion is unique up to a multiplicative constant, and that the behavior of any other linearly independent solution of the recursion, say $y_{n}(z)$, is essentially determined by the behavior of a dominant solution, in the sense that $\left|y_{n}(z) / g_{n}(z)\right| \sim B$ as $n \rightarrow \infty$, $B \neq 0$ and not depending on $n$. Because of this property, a generic solution of (1.1), which can be written as $y_{n}(z)=A f_{n}(z)+B g_{n}(z)$, where $A$ and $B$ do not depend on $n$, will always behave as a dominant solution, unless $B=0$. Since this last condition is normally not fulfilled in numerical computation (if only because of round-off errors), then the error when trying to compute the minimal solution $f_{n}(z)$ by using the recursion in the forward direction (increasing $n$ ) becomes unacceptably large.

The construction of numerically satisfactory pairs and the numerical behavior explained in the last paragraph underscore the importance of identifying minimal solutions (in both directions, increasing and decreasing $n$ ) before using a recursion for computational purposes. In the case of recurrence relations for special functions, several cases have already been analysed; see for example the references [3] and [8]. More recently, Gauss hypergeometric cases have been examined in detail in [5], 6], and [4, Ch. 4].

In this paper we analyse dominant and minimal solutions of confluent hypergeometric recursions, revising several cases already present in the literature, and also completing the simplest directions of recursion. The structure of the paper is as follows. First we briefly recall properties of Kummer and Whittaker functions, including the notation that we will use for the recursions and the connection formulas that will allow us to reduce the number of cases to be analysed. In each of these situations we will give minimal and dominant solutions (when they exist) for complex values of the variable $z$ in the principal sector in $|\operatorname{ph} z|<\pi$. For obtaining this information we will make use of Perron's theorem, in the formulation given in [8] and [4, §4.3], together with asymptotic estimations for Whittaker functions with large values of the parameters from [2]. For the sake of clarity, these estimates are grouped in the Appendix and are referred to when needed.

\section{The Kummer and Whittaker functions}

2.1. The Kummer functions. The Kummer differential equation, also called confluent hypergeometric differential equation, is given by

$$
z \frac{d^{2}}{d z^{2}} w(z)+(c-z) \frac{d}{d z} w(z)-a w(z)=0 .
$$

One solution of this equation is the confluent hypergeometric function of the first kind 


$$
w_{1}(z)={ }_{1} F_{1}(a ; c ; z)=\sum_{k=0}^{\infty} \frac{(a)_{k}}{(c)_{k}} \frac{z^{k}}{k !}
$$

where $(a)_{k}$ is the Pochhammer symbol, $(a)_{k}=\Gamma(a+k) / \Gamma(a), k=1,2, \ldots$, with $(a)_{0}=1$. Another solution is the confluent hypergeometric function of the second kind $w_{2}(z)=U(a, c, z)$. These two functions are linearly independent when $a \neq$ $0,-1,-2, \ldots$.

Other solutions of (2.1) are

$$
\begin{aligned}
w_{3}(z) & =z^{1-c}{ }_{1} F_{1}(a+1-c ; 2-c ; z), \\
w_{4}^{ \pm}(z) & =e^{z} U\left(c-a, c, z e^{ \pm \pi i}\right) .
\end{aligned}
$$

These five solutions are related by the following connection formulas; see [1, p. 504] and [7, $\S \S 1.9$ and 2.2.2]:

$$
\begin{aligned}
U(a, c, z) & =\frac{\Gamma(1-c)}{\Gamma(a+1-c)}{ }_{1} F_{1}(a ; c ; z)+\frac{\Gamma(c-1)}{\Gamma(a)} z^{1-c}{ }_{1} F_{1}(a+1-c ; 2-c ; z), \\
{ }_{1} F_{1}(a ; c ; z) & =\frac{e^{ \pm \pi i a} \Gamma(c)}{\Gamma(c-a)} U(a, c, z)+\frac{e^{ \pm \pi i(a-c)+z} \Gamma(c)}{\Gamma(a)} U\left(c-a, c, z e^{\mp \pi i}\right) .
\end{aligned}
$$

It is worth noting that ${ }_{1} F_{1}(a ; c ; z)$ is in general not defined when $c=0,-1,-2, \ldots$, due to the definition of the Pochhammer symbol. However, the function $U(a, c, z)$ is well defined in the first relation of (2.4) for any values of $a$ and $c$, as can be verified taking limits $c \rightarrow-m, m$ an integer, when necessary.

Other functional identities that will be used later are [1]

$$
\begin{aligned}
& { }_{1} F_{1}(a ; c ; z)=e^{z}{ }_{1} F_{1}(c-a ; c ;-z), \\
& U(a, c, z)=z^{1-c} U(a+1-c, 2-c, z), \\
& z^{1-c}{ }_{1} F_{1}(a+1-c ; 2-c ; z)=z^{1-c} e^{z}{ }_{1} F_{1}(1-a ; 2-c ;-z), \\
& e^{z} U\left(c-a, c, z e^{ \pm \pi i}\right)=z^{1-c} e^{z \pm \pi i(1-b)} U\left(1-a, 2-c, z e^{ \pm \pi i}\right) .
\end{aligned}
$$

2.2. The Whittaker functions. The Whittaker functions are denoted by $M_{\kappa, \mu}(z)$ and $W_{\kappa, \mu}(z)$ and are the standard solutions of the Whittaker differential equation

$$
\frac{d^{2}}{d z^{2}} w(z)=\left(\frac{1}{4}-\frac{\kappa}{z}+\frac{\mu^{2}-\frac{1}{4}}{z^{2}}\right) w(z) .
$$

This equation is the transformation of the Kummer equation (2.1) to normal form. The parameters $\kappa$ and $\mu$ are related to the parameters $a$ and $c$ of the Kummer functions by the following formulas:

$$
\kappa=\frac{1}{2}(c-2 a), \quad \mu=\frac{1}{2}(c-1) .
$$

The Whittaker functions $M_{\kappa, \mu}(z)$ and $W_{\kappa, \mu}(z)$ can be expressed in terms of the Kummer functions. We have

$$
\begin{aligned}
& M_{\kappa, \mu}(z)=e^{-z / 2} z^{1 / 2+\mu} F_{1}\left(\frac{1}{2}+\mu-\kappa ; 1+2 \mu ; z\right), \\
& W_{\kappa, \mu}(z)=e^{-z / 2} z^{1 / 2+\mu} U\left(\frac{1}{2}+\mu-\kappa, 1+2 \mu, z\right) .
\end{aligned}
$$

Conversely,

$$
\begin{aligned}
& { }_{1} F_{1}(a ; c ; z)=e^{z / 2} z^{-c / 2} M_{c / 2-a, c / 2-1 / 2}(z), \\
& U(a, c, z)=e^{z / 2} z^{-c / 2} W_{c / 2-a, c / 2-1 / 2}(z) .
\end{aligned}
$$


Other solutions of the Whittaker equation are

$$
\begin{aligned}
M_{-\kappa, \mu}\left(z e^{ \pm \pi i}\right) & =e^{ \pm(\mu+1 / 2) \pi i} M_{\kappa, \mu}(z), \\
W_{\kappa,-\mu}(z) & =W_{\kappa, \mu}(z) .
\end{aligned}
$$

\section{Confluent hypergeometric ReCursions}

When considering three-term recursions for confluent hypergeometric functions, we will take fixed values of the parameters $a$ and $c$ and will introduce a dependence on an integer parameter $n$. In other words, we will consider confluent hypergeometric functions with the following notation:

$$
{ }_{1} F_{1}\left(a+\epsilon_{1} n ; c+\epsilon_{2} n ; z\right), \quad U\left(a+\epsilon_{1} n, c+\epsilon_{2} n, z\right),
$$

where $\epsilon_{i}=0, \pm 1\left(\epsilon_{1}=\epsilon_{2}=0\right.$ excluded $)$, that satisfy a three-term recurrence relation of the form (1.1).

Naturally, the choice of $\epsilon_{1}$ and $\epsilon_{2}$ will produce different directions of recursion, that we will denote by pairs $\left(\operatorname{sign}\left(\epsilon_{1}\right), \operatorname{sign}\left(\epsilon_{2}\right)\right)$. For example, when $\epsilon_{1}=1$ and $\epsilon_{2}=0$, the recursion is denoted by $(+0)$. The different choices of $\epsilon_{i}$ produce eight recursions. In the next sections we will give the minimal solution in each case, together with the region of the complex plane where it is minimal.

Remarks.

(1) It is worth noting that in order to have a recursion satisfied by both Kummer functions (3.1) it is necessary to include additional factors (usually ratios of gamma functions), which can be obtained from the connection formulas (2.4). In all cases (except $\epsilon_{1}=\epsilon_{2}=-1$ ) we will take as the first solution of the recursions the function ${ }_{1} F_{1}\left(a+\epsilon_{1} n ; c+\epsilon_{2} n ; z\right)$, and derive the rest from (2.4).

(2) When selecting the gamma functions in front of the solutions of recurrence relations, we avoid gamma functions with negative $n$ by using the relation

$$
\Gamma(z-n)=\frac{(-1)^{n} \pi}{\sin (\pi z) \Gamma(n+1-z)},
$$

and by neglecting those factors in this relation that do not depend on $n$.

(3) We will not insist on treating the case $c=0,-1,-2, \ldots$ and the problems in the definition of the function ${ }_{1} F_{1}(a ; c ; z)$ separately. In this case one can take other solutions using (2.4), or if the ${ }_{1} F_{1}$ function is the minimal solution then rescale everything and use ${ }_{1} F_{1}(a ; c ; z) / \Gamma(c)$.

(4) In principle we could consider other values of $\epsilon_{i}$, but the recursions become increasingly more complicated. It is of interest to observe, however, that for other specific cases simple and important recursions arise. For example, for ${ }_{1} F_{1}(a+n ; c+2 n ; z)$, with special values of $a$ and $c$, this Kummer function becomes a known special function. When $a=\frac{1}{2} c$ it reduces to a Bessel function, and when $a=L-i \eta$ and $c=2 L$ it becomes a Coulomb wave function (see [1, Chapters 9 and 14]). The functions ${ }_{1} F_{1}\left(\frac{1}{2} a+\frac{1}{2} n ; \frac{1}{2} \pm \frac{1}{4} ; z\right)$ are related to parabolic cylinder functions (see [1, Ch. 19]). 


\section{The $(+0)$ RECURSION}

The parameters of the three-term recursion (1.1) with respect to $a$ are

$$
a_{n}=\frac{a+n-c}{a+n}, \quad b_{n}=-\frac{2 a+2 n-c+z}{a+n} .
$$

These coefficients have the following asymptotic behavior:

$$
a_{n} \sim 1, \quad b_{n} \sim-2, \quad n \rightarrow \infty,
$$

and it follows from Perron's theorem (see [8, Theorem 13.1, Case 2] and [4, §4.3] that

$$
\limsup _{n \rightarrow \infty}\left|y_{n}(z)\right|=1
$$

for each non-trivial solution of the recursion.

Four different solutions of the recursion are

$$
\begin{aligned}
& y_{n}^{(1)}(z)={ }_{1} F_{1}(a+n ; c ; z), \\
& y_{n}^{(2)}(z)=\Gamma(a+1-c+n) U(a+n, c, z), \\
& y_{n}^{(3 \pm)}(z)=\frac{(-1)^{n}}{\Gamma(a+n)} U\left(c-a-n, c, z e^{ \pm \pi i}\right) .
\end{aligned}
$$

Using the Whittaker notation, the parameters are (see (2.7))

$$
\kappa=\frac{1}{2}(c-2 a-2 n), \quad \mu=\frac{1}{2}(c-1) .
$$

Since $\kappa<0$ for large $n$, the asymptotic relations in (9.3) (9.6) cannot be applied straightforwardly. However, we have several relations between these functions that are useful when identifying the minimal and dominant solutions of the recursion.

4.1. The solution $y_{n}^{(1)}(z)$. This solution is related to the Whittaker function $M_{\kappa, \mu}(z)$. In order to reverse the sign of $\kappa$ we use the relation (see (2.10)

$$
M_{\kappa, \mu}(z)=e^{\mp(\mu+1 / 2) \pi i} M_{-\kappa, \mu}\left(z e^{ \pm \pi i}\right) .
$$

It follows that we can use the asymptotic relation given in (9.3) for the solution $y_{n}^{(1)}(z)$, when we replace $z$ with $z e^{ \pm \pi i}$. We will choose the plus sign whenever $-\pi<\operatorname{ph} z \leq 0$ and the minus sign when $0<\operatorname{ph} z \leq \pi$; hence, the principal argument is always maintained.

The $J$-Bessel function in the asymptotic relation (9.3) has argument $2 \sqrt{\kappa z}$, since the quantity $c(\alpha)$ in (9.10) and (9.12) has the following behavior (see also [2, Eq. (3.11)])

$$
c(\alpha)=4-\frac{1}{6} \alpha^{2}+\mathcal{O}\left(\alpha^{4}\right), \quad \alpha \rightarrow 0 .
$$

Therefore, the $J$-Bessel function has its argument in the right half-plane (see (9.12) ), and it can be written in terms of the modified $I$-Bessel function, because of the relation

$$
J_{\nu}\left(w e^{ \pm \frac{1}{2} \pi i}\right)=e^{\mp \frac{1}{2} \nu \pi i} I_{\nu}(w), \quad-\frac{1}{2} \pi<\operatorname{ph} w \leq \frac{1}{2} \pi,
$$

which follows from [1, Eq. 9.6.3]. Since the modified Bessel function $I_{\nu}(w)$ is exponentially large as $\Re w \rightarrow+\infty$, we conclude that the solution $y_{n}^{(1)}(z)$ is not a candidate for the minimal solution of the recursion. 
The approximations for this solution can also be obtained from known estimates in the literature. From [7, p. 80] it follows that

$$
M_{-\kappa, \mu}(z) \sim \sqrt{z} \Gamma(2 \mu+1) \kappa^{-\mu} I_{2 \mu}(2 \sqrt{\kappa z}), \quad \kappa \rightarrow \infty
$$

in the sector $-\pi<\operatorname{ph} z<\pi$, or in terms of the Kummer ${ }_{1} F_{1}$-function,

$$
{ }_{1} F_{1}(a+n ; c ; z) \sim \Gamma(c)(n z)^{(1-c) / 2} e^{z / 2} I_{c-1}(2 \sqrt{n z}), \quad n \rightarrow \infty .
$$

4.2. The solution $y_{n}^{(2)}(z)$. This solution is the minimal solution for $z$ inside the sector $-\pi<\operatorname{ph} z<\pi$. This follows from the estimates given in the Appendix, but we can also use the analogs of the estimates in (4.9)-(4.10). From [7, p. 81] it follows that we have, in terms of the modified $K$-Bessel function,

$$
W_{-\kappa, \mu}(z) \sim \frac{2 \sqrt{z} \kappa^{\mu}}{\Gamma\left(\frac{1}{2}+\mu+\kappa\right)} K_{2 \mu}(2 \sqrt{\kappa z}), \quad \kappa \rightarrow \infty
$$

in the sector $-\pi<\operatorname{ph} z<\pi$, or in terms of the Kummer $U$-function,

$$
\Gamma(a+n+1-c) U(a+n, c, z) \sim 2(n z)^{\frac{1-c}{2}} e^{z / 2} K_{c-1}(2 \sqrt{n z}), \quad n \rightarrow \infty .
$$

The function $K_{\nu}(w)$ is exponentially small as $\Re w \rightarrow+\infty$, and we conclude that

$$
\lim _{n \rightarrow \infty} \frac{y_{n}^{(2)}(z)}{y_{n}^{(1)}(z)}=0, \quad-\pi<\operatorname{ph} z<\pi,
$$

from which it follows that the solution $y_{n}^{(2)}(z)$ is the minimal solution of this recursion for all non-negative $z$.

\section{The $(-0)$ RECURSION}

In this case the coefficients can be obtained directly from the $(+0)$ recursion

$$
a_{n}=\frac{n-a}{c-a+n}, \quad b_{n}=\frac{2 a-2 n-c+z}{c-a+n} .
$$

These coefficients have the following asymptotic behavior:

$$
a_{n} \sim 1, \quad b_{n} \sim-2, \quad n \rightarrow \infty,
$$

and it follows from Perron's theorem (see [8, Theorem 13.1, Case 2] and [4, §4.3] that

$$
\limsup _{n \rightarrow \infty}\left|y_{n}(z)\right|=1,
$$

for each non-trivial solution of the recursion.

Four different solutions are

$$
\begin{aligned}
& y_{n}^{(1)}(z)={ }_{1} F_{1}(a-n ; c ; z), \\
& y_{n}^{(2)}(z)=\frac{(-1)^{n}}{\Gamma(c-a+n)} U(a-n, c, z), \\
& y_{n}^{(3 \pm)}(z)=\Gamma(1-a+n) U\left(c-a+n, c, z e^{ \pm \pi i}\right),
\end{aligned}
$$

which follow from the connection formula (2.4).

The information given by Perron's theorem in (5.3) is not sufficient to identify the minimal and dominant solutions. The asymptotic behavior for $n \rightarrow+\infty$ follows from the large $\kappa$ behavior of the Whittaker functions given in (9.3)-(9.6), since the Whittaker parameters are

$$
\kappa=\frac{1}{2}(c-2 a+2 n), \quad \mu=\frac{1}{2}(c-1) .
$$


In principle it is possible to analyse the behavior of the solutions $y_{n}^{(1)}(z)$ and $y_{n}^{(2)}(z)$ by using these parameters, together with the approximations (9.3) and (9.4), without any change of sign in the parameters. However, there is a simpler way to identify the minimal solution: we use the known asymptotic behavior of the Hankel functions in (9.5) and (9.6). When the argument $w$ is large we have (see [1, Eqs. (9.2.3) and (9.2.4)])

$$
\begin{array}{ll}
H_{\nu}^{(1)}(w) \sim \sqrt{\frac{2}{\pi w}} e^{i\left(w-\frac{1}{2} \nu \pi-\frac{1}{4} \pi\right)}, & -\pi<\operatorname{ph} w<2 \pi, \\
H_{\nu}^{(2)}(w) \sim \sqrt{\frac{2}{\pi w}} e^{-i\left(w-\frac{1}{2} \nu \pi-\frac{1}{4} \pi\right)}, \quad-2 \pi<\operatorname{ph} w<\pi .
\end{array}
$$

As before, the argument of the Hankel functions in (9.5) and (9.6) is $2 \sqrt{\kappa z}$, now with positive $\kappa$.

Noting that the $J-$ and $Y$-Bessel functions in (9.3) and (9.4) are linear combinations of the Hankel functions, we see that they are exponentially large whenever $2 \sqrt{\kappa z}$ is not real. This exponential growth means that the solutions $y_{n}^{(1)}(z)$ and $y_{n}^{(2)}(z)$ are not minimal when $\Im z \neq 0$, because, as we discuss next, the minimal solution decreases exponentially.

Observe that when $z$ belongs to the upper half-plane, the Hankel function $H_{\nu}^{(1)}(2 \sqrt{\kappa z})$ present in (9.5) becomes exponentially small for large values of $\kappa z$, and when $z$ belongs to the lower half-plane, the Hankel function $H_{\nu}^{(2)}(2 \sqrt{\kappa z})$ present in (9.6) becomes exponentially small for large values of $\kappa z$. Then, using the relations between the Whittaker functions and the Kummer functions given in (2.8) and (2.9), together with (9.5) and (9.6), we conclude that when $z$ is in the upper half-plane, the solution

$$
y_{n}^{(3-)}(z)=\Gamma(1-a+n) U\left(c-a+n, c, z e^{-\pi i}\right)
$$

is the unique minimal solution of the recurrence relation, whereas if $z$ is in the lower half-plane, then

$$
y_{n}^{(3+)}(z)=\Gamma(1-a+n) U\left(c-a+n, c, z e^{\pi i}\right)
$$

is the minimal solution.

When $z$ is not real both $y_{n}^{(1)}(z)$ and $y_{n}^{(2)}(z)$ are dominant solutions. On the negative real axis $y_{n}^{(3-)}(z)$ is the minimal solution when we take $\operatorname{ph} z=+\pi$ and $y_{n}^{(3+)}(z)$ is minimal when $\operatorname{ph} z=-\pi$. The other solutions $y_{n}^{(1)}(z)$ and $y_{n}^{(2)}(z)$ are dominant solutions on the negative real axis.

On the positive real axis there is no minimal solution. All Bessel and Hankel functions have positive arguments, and none of the four solutions in (5.4) is exponentially decreasing when $z>0$.

We observe that for $a=0,-1,-2, \ldots$ the solutions $y_{n}^{(1)}(z)$ and $y_{n}^{(2)}(z)$ are linearly dependent, in fact, they reduce to polynomials and are oscillating for $c>0$ and $z>0$. This known behavior also follows from the relation with the Laguerre polynomials ([8, p. 190])

$$
L_{n}^{(\alpha)}(z)=\frac{(-1)^{n}}{n !} U(-n, \alpha+1, z)=\left(\begin{array}{c}
n+\alpha \\
n
\end{array}\right){ }_{1} F_{1}(-n ; \alpha+1 ; z) .
$$




\section{The $(-+)$ AND $(+-)$ RECURSIONS}

6.1. The $(-+)$ recursion. The coefficients of the recursion (1.1) are given by

$$
\begin{aligned}
a_{n}= & (c+n-1)(c+n)(-a+n)(-n+a+z-1) / d_{n}, \\
b_{n}= & (c+n)\left(n^{3}+(3 z+c-2 a) n^{2}\right. \\
& +\left(3 z+c-6 a z+a^{2}-5 z^{2}+a-2 a c-1\right) n+z^{3} \\
& \left.+z^{2}(4 a-c-1)+3 a z(a-1)+a(a c-c-a+1)\right) / d_{n}, \\
d_{n}= & z(c+2 n+1-a)(c+2 n-a)(-n+z+a) .
\end{aligned}
$$

We have

$$
a_{n} \sim \frac{n}{4 z}, \quad b_{n} \sim-\frac{n}{4 z}, \quad n \rightarrow \infty .
$$

The application of Perron's theorem (see [8, Theorem 13.1, Case 1] and [4, §4.3]) yields the following behavior of the solutions:

$$
\frac{f_{n+1}(z)}{f_{n}(z)} \sim 1, \quad \frac{g_{n+1}(z)}{g_{n}(z)} \sim \frac{n}{4 z}, \quad n \rightarrow \infty,
$$

the solution $f_{n}$ being minimal. If $a-n \neq 0,-1,-2, \ldots$, then two independent solutions of the recursion are the Kummer functions

$$
\begin{aligned}
& y_{n}^{(1)}(z)={ }_{1} F_{1}(a-n ; c+n ; z), \\
& y_{n}^{(2)}(z)=\frac{(-1)^{n} \Gamma(c+n)}{\Gamma(c-a+2 n)} U(a-n, c+n, z) .
\end{aligned}
$$

Bearing in mind the connection between the Kummer and Whittaker functions given in (2.8) and (2.9), we see that the corresponding Whittaker functions have parameters

$$
\kappa=\frac{1}{2}(c-2 a+3 n), \quad \mu=\frac{1}{2}(c+n-1),
$$

and it is clear that the shift $n \rightarrow n+1$ is equivalent to $\kappa \rightarrow \kappa+3 / 2$ and $\mu \rightarrow \mu+1 / 2$.

6.1.1. Asymptotic estimates. When $n \rightarrow \infty$ both $\kappa$ and $\mu$ tend to $\infty$, so in order to describe the behavior of the Whittaker functions when $n \rightarrow \infty$ we will need asymptotic expansions for large $\kappa$ that hold uniformly with respect to $\mu$. Dunster's expansions summarized in (9.3)-(9.6) can also be used in the present case.

The parameter $\xi$ of (9.1) is again small for fixed $z$, and from (6.5) we conclude that $\alpha \rightarrow 2 / 3$ when $n$ is large. The quantity $\zeta$ used in the asymptotic estimates is small because $|\xi|$ is small, and we have the same behavior as in (9.10):

$$
\zeta=c(\alpha) \xi+\mathcal{O}\left(\xi^{2}\right), \quad \xi \rightarrow 0 .
$$

In the present case $\alpha$ does not tend to 0 ; using $\kappa / \mu \rightarrow 3$ we obtain

$$
c(\alpha)=\frac{32}{3 e}+\mathcal{O}\left(n^{-1}\right), \quad n \rightarrow \infty .
$$

Since $\xi=\mathcal{O}\left(n^{-1}\right)$ for fixed $z$, then

$$
\zeta=\frac{32}{3 e} \xi+\mathcal{O}\left(\xi^{2}\right), \quad \xi \rightarrow 0 .
$$


With this estimate, the argument of the Bessel functions that appear in the asymptotic expansions (9.3)-(9.6) can be taken as

$$
\kappa \sqrt{\zeta} \sim \kappa \sqrt{\frac{32}{3 e} \frac{z}{\kappa}}=\sqrt{\frac{32}{3 e} \kappa z} .
$$

6.1.2. The solution $y_{n}^{(1)}(z)$. To assign the minimal solution of this recursion, we concentrate on the asymptotic expansion for $M_{\kappa, \mu}(z)$ given in (9.3). We will estimate the following ratio (see also (6.5) ):

$$
R_{\kappa, \mu}(z)=\frac{M_{\kappa+3 / 2, \mu+1 / 2}(z)}{M_{\kappa, \mu}(z)}
$$

for large values of $\kappa$ and $\mu$, using $\kappa / \mu \rightarrow 3$. The relevant factor in the asymptotic behavior of $M_{\kappa, \mu}(z)$ is (see (9.3) and also (9.8) and (9.9))

$$
\widetilde{F}_{\kappa, \mu}(z) J_{2 \mu}(\kappa \sqrt{\zeta})
$$

where

$$
\widetilde{F}_{\kappa, \mu}(z)=e^{\mu} \Gamma(2 \mu+1) \Psi_{\kappa, \mu},
$$

and $\Psi_{\kappa, \mu}$ is given in (9.7).

A long but straightforward computation shows that

$$
\frac{\widetilde{F}_{\kappa+3 / 2, \mu+1 / 2}(z)}{\widetilde{F}_{\kappa, \mu}(z)} \sim \frac{1}{2} \sqrt{n}, \quad n \rightarrow \infty .
$$

In order to estimate the Bessel function in (6.11) we note that in this case both the order and the argument are large, so we will use an asymptotic estimation of this Bessel function for large order which holds uniformly with respect to the argument. This can be accomplished by means of the Airy-type approximation [1, Eq. 9.3.6]

$$
J_{\nu}(\nu w)=\left(\frac{4 \chi}{1-w^{2}}\right)^{1 / 4}\left[\frac{\operatorname{Ai}\left(\nu^{2 / 3} \chi\right)}{\nu^{1 / 3}}+\frac{\exp \left(-\frac{2}{3} \nu \chi^{3 / 2}\right)}{1+\nu^{1 / 6}|\chi|^{1 / 4}} \mathcal{O}\left(\nu^{-4 / 3}\right)\right],
$$

which holds for large values of $\nu$, uniformly for $-\pi<\operatorname{ph} z<\pi$. Here the variable $\chi$ is defined as in [1, Eq. 9.3.38]:

$$
\frac{2}{3} \chi^{3 / 2}=\log \frac{1+\sqrt{1-w^{2}}}{w}-\sqrt{1-w^{2}} .
$$

In the present situation, if we set $\beta=32 /(3 e)$, our Bessel function reads

$$
J_{2 \mu}(\sqrt{\beta \kappa z})=J_{2 \mu}\left(2 \mu \frac{\sqrt{\beta \kappa z}}{2 \mu}\right) .
$$

Therefore, we use the parameters

$$
\nu=2 \mu, \quad w=\frac{\sqrt{\beta \kappa z}}{2 \mu},
$$

and we see that the argument of the Airy function in (6.14) is large when $\mu$ is large, which allows us to use an asymptotic estimate for this function. Using [1, Eq. 10.4.59] we obtain

$$
\operatorname{Ai}\left(\nu^{2 / 3} \chi\right)=\frac{1}{2} \pi^{-1 / 2} \nu^{-1 / 6} \chi^{-1 / 4} e^{-\frac{2}{3} \nu \chi^{3 / 2}}\left(1+\mathcal{O}\left(\nu^{-1}\right)\right) .
$$


It follows that we can estimate (6.14) in the form

$$
J_{\nu}(\nu w) \sim\left(1-w^{2}\right)^{-1 / 4} \frac{1}{\sqrt{2 \pi \nu}} e^{-\frac{2}{3} \nu \chi^{3 / 2}}, \quad \nu \rightarrow \infty .
$$

Since our objective is to estimate the ratio of two Whittaker functions, with parameters $\kappa+3 / 2, \mu+1 / 2$ and $\kappa, \mu$ respectively, we define the following shifted variables:

$$
\widetilde{w}=\frac{\sqrt{\beta(\kappa+3 / 2) z}}{2 \mu+1}, \quad \frac{2}{3} \widetilde{\chi}^{3 / 2}=\log \frac{1+\sqrt{1-\widetilde{w}^{2}}}{\widetilde{w}}-\sqrt{1-\widetilde{w}^{2}} .
$$

Thus the ratio of Bessel functions satisfies

$$
\frac{J_{2 \mu+1}((2 \mu+1) \widetilde{w})}{J_{2 \mu}(2 \mu w)} \sim\left(\frac{1-\widetilde{w}^{2}}{1-w^{2}}\right)^{-1 / 4}\left(1-\frac{1}{2 \mu+1}\right)^{1 / 2} \frac{e^{-\frac{2}{3}(2 \mu+1) \tilde{\chi}^{3 / 2}}}{e^{-\frac{2}{3} 2 \mu \chi^{3 / 2}}} .
$$

The first two terms of this approximation tend to 1 when $n \rightarrow \infty$, and it can be verified that

$$
\frac{e^{-\frac{2}{3}(2 \mu+1) \tilde{\chi}^{3 / 2}}}{e^{-\frac{2}{3} 2 \mu \chi^{3 / 2}}} \sim 2 \sqrt{\frac{z}{n}}, \quad n \rightarrow \infty
$$

Hence,

$$
R_{\kappa, \mu}(z)=\frac{M_{\kappa+3 / 2, \mu+1 / 2}(z)}{M_{\kappa, \mu}(z)} \sim \sqrt{z}, \quad n \rightarrow \infty .
$$

Taking into account the first relation in (2.8), that is,

$$
M_{\kappa, \mu}(z)=e^{-z / 2} z^{1 / 2+\mu}{ }_{1} F_{1}\left(\frac{1}{2}+\mu-\kappa ; 1+2 \mu ; z\right),
$$

it follows that

$$
R_{\kappa, \mu}(z)=\frac{M_{\kappa+3 / 2, \mu+1 / 2}(z)}{M_{\kappa, \mu}(z)}=\sqrt{z} \frac{{ }_{1} F_{1}\left(-\frac{1}{2}+\mu-\kappa ; 2+2 \mu ; z\right)}{{ }_{1} F_{1}\left(\frac{1}{2}+\mu-\kappa ; 1+2 \mu ; z\right)} .
$$

This finally gives

$$
\frac{{ }_{1} F_{1}\left(-\frac{1}{2}+\mu-\kappa ; 2+2 \mu ; z\right)}{{ }_{1} F_{1}\left(\frac{1}{2}+\mu-\kappa ; 1+2 \mu ; z\right)} \sim 1, \quad n \rightarrow \infty,
$$

and expressing $\kappa$ and $\mu$ in terms of $a, c$ and $n$ it follows that the Kummer function ${ }_{1} F_{1}(a-n ; c+n ; z)$ is the minimal solution of the $(-+)$ recursion when $n \rightarrow \infty$ for all complex $z$. The solution $y_{n}^{(2)}(z)$ related to the other Kummer function in (6.4) is a dominant solution for all $z$.

6.2. The $(+-)$ recursion. In this case the coefficients are

$$
\begin{aligned}
a_{n}= & -z(2 n+a-c)(2 n-1+a-c)(n+a+z) / d_{n}, \\
b_{n}= & (n-c)\left(-n^{3}+(-2 a+3 z+c) n^{2}\right. \\
& +\left(2 a c-a^{2}+1-a-c+5 z^{2}+6 a z-3 z\right) n+z^{3} \\
& \left.+z^{2}(4 a-c-1)+3 a z(a-1)+a(a c-a-c+1)\right) / d_{n}, \\
d_{n}= & (n-c+1)(-c+n)(a+n)(n+a+z-1),
\end{aligned}
$$

and they satisfy

$$
a_{n} \sim-\frac{4 z}{n}, \quad b_{n} \sim-1, \quad n \rightarrow \infty
$$


The application of Perron's theorem (see [8, Theorem 13.1, Case 1] and [4, §4.3]) yields the following behavior of the solutions:

$$
\frac{f_{n+1}(z)}{f_{n}(z)} \sim-\frac{4 z}{n}, \quad \frac{g_{n+1}(z)}{g_{n}(z)} \sim 1, \quad n \rightarrow \infty,
$$

the solution $f_{n}$ being minimal. Two independent solutions of (6.27) are the Kummer functions

$$
\begin{aligned}
& y_{n}^{(1)}(z)={ }_{1} F_{1}(a+n ; c-n ; z), \\
& y_{n}^{(2)}(z)=\frac{\Gamma(a+1-c+2 n)}{\Gamma(1-c+n)} U(a+n, c-n, z) .
\end{aligned}
$$

A third solution can be constructed using the connection formula (2.4) and reads (6.31)

$$
y_{n}^{(3)}(z)=\frac{\Gamma(a+1-c+2 n) \Gamma(c-n-1)}{\Gamma(a+n) \Gamma(1-c+n)} z^{1-c+n}{ }_{1} F_{1}(a+1-c+2 n ; 2-c+n ; z) .
$$

If we use Whittaker notation, then we can rewrite this last solution as

$$
y_{\kappa, \mu}^{(3)}(z)=\frac{\Gamma(\mu+\kappa+1 / 2) \Gamma(\mu-\kappa+1)}{\Gamma(2 \mu) \Gamma(2 \mu+1)} e^{z / 2} z^{\mu-1 / 2} M_{-\kappa, \mu}(z),
$$

where

$$
\kappa=\frac{1}{2}(-c+2 a+3 n), \quad \mu=\frac{1}{2}(-c+n+1) .
$$

We use the first line of (2.10), that is,

$$
M_{-\kappa, \mu}(z)=e^{ \pm(\mu+1 / 2) \pi i} M_{\kappa, \mu}\left(z e^{ \pm \pi i}\right),
$$

again choosing the plus or minus sign depending on the phase of $z$. Now the asymptotic behavior of the function $M_{\kappa, \mu}\left(z e^{ \pm \pi i}\right)$ can be analysed using the results from the $(-+)$ recursion, since both $\kappa$ and $\mu$ are positive and show the same dependence on $n$ as in that case. Thus, using (6.23) we obtain

$$
\frac{y_{\kappa+3 / 2, \mu+1 / 2}^{(3)}(z)}{y_{\kappa, \mu}^{(3)}(z)} \sim-\frac{6 z}{\kappa},
$$

which, writing $\kappa$ and $\mu$ in terms of $n$, gives

$$
\frac{y_{n+1}^{(3)}(z)}{y_{n}^{(3)}(z)} \sim-\frac{4 z}{n}
$$

Hence, the function $y_{n}^{(3)}(z)$ is the minimal solution of the $(+-)$ recursion, in accordance with (6.29). It can be written in terms of the other two solutions $y_{n}^{(1)}(z)$ and $y_{n}^{(2)}(z)$, which are dominant:

$$
y_{n}^{(3)}(z)=\frac{\Gamma(a+1-c+2 n)}{\Gamma(1-c+n)} U(a+n, c-n, z)-{ }_{1} F_{1}(a+n ; c-n ; z) .
$$




\section{The $(++)$ AND RELATED RECURSIONS}

7.1. The $(++)$ recursion. In this case the coefficients of the recursion (1.1) read

$$
a_{n}=-\frac{(c+n)(c+n-1)}{(a+n) z}, \quad b_{n}=\frac{(c+n)(c+n-1-z)}{(a+n) z} .
$$

Therefore,

$$
a_{n} \sim-\frac{n}{z}, \quad b_{n}=\frac{n}{z}, \quad n \rightarrow \infty,
$$

and Perron's theorem establishes the following behavior of the solutions

$$
\frac{f_{n+1}(z)}{f_{n}(z)} \sim 1, \quad \frac{g_{n+1}(z)}{g_{n}(z)} \sim-\frac{n}{z}, \quad n \rightarrow \infty,
$$

the solution $f_{n}(z)$ being minimal.

Four solutions of this recursion are

$$
\begin{aligned}
& y_{n}^{(1)}(z)={ }_{1} F_{1}(a+n ; c+n ; z), \\
& y_{n}^{(2)}(z)=(-1)^{n} \Gamma(c+n) U(a+n, c+n, z), \\
& y_{n}^{(3 \pm)}(z)=\frac{\Gamma(c+n)}{\Gamma(a+n)} U\left(c-a, c+n, z e^{ \pm \pi i}\right),
\end{aligned}
$$

which follows from (2.4).

In order to determine the asymptotic behavior of these solutions we first observe that the solution $y_{n}^{(1)}(z)$ reduces to the exponential function $e^{z}$ when $a=c$ and all complex $z$. Also, by using the first line in (2.5) it follows that

$$
y_{n}^{(1)}(z)=e^{z}{ }_{1} F_{1}(c-a ; c+n ;-z),
$$

and using the series expansions in (2.2) we see that

$$
y_{n}^{(1)}(z)=e^{z}[1+\mathcal{O}(1 / n)], \quad n \rightarrow \infty,
$$

uniformly for all bounded $z$. Therefore, in view of (7.3) we infer that $y_{n}^{(1)}(z)$ of (17.4) is the minimal solution.

The asymptotic analysis of $y_{n}^{(2)}(z)$ is also quite simple in this case. From the integral representation (see [1, p. 505]),

$$
U(a+n, c+n, z)=\frac{1}{\Gamma(a+n)} \int_{0}^{\infty} e^{-z t} t^{a+n-1}(1+t)^{c-a-1} d t,
$$

where $\Re a>0, \Re z>0$. This type of Laplace integral is discussed in [9], and it follows from that paper that

$$
U(a+n, c+n, z)=z^{-a-n}[1+\mathcal{O}(1 / n)], \quad n \rightarrow \infty .
$$

From [9, §3.3] it follows that this estimate holds for bounded complex values of $z$ with $|\mathrm{ph} z|<\frac{1}{2} \pi$. The second relation in (2.4) can be used for extending (7.8) to the sector $|\operatorname{ph} z|<\pi$. This shows that $y_{n}^{(2)}(z)$ corresponds to the function $g_{n}(z)$ in (7.3), which is a dominant solution. 
7.2. The $(0+)$ recursion. In this case the coefficients of the recursion are (see [1, Eq. 13.4.2])

$$
a_{n}=\frac{(c+n)(c+n-1)}{z(c+n-a)}, \quad b_{n}=\frac{(c+n)(1-c-n-z)}{z(c+n-a)} .
$$

Therefore,

$$
a_{n} \sim \frac{n}{z}, \quad b_{n}=-\frac{n}{z},
$$

and according to Perron's theorem the solutions satisfy

$$
\frac{f_{n+1}(z)}{f_{n}(z)} \sim 1, \quad \frac{g_{n+1}(z)}{g_{n}(z)} \sim \frac{n}{z},
$$

the solution $f_{n}(z)$ being minimal.

Four solutions of this recursion are

$$
\begin{aligned}
& y_{n}^{(1)}(z)={ }_{1} F_{1}(a ; c+n ; z), \\
& y_{n}^{(2)}(z)=\frac{\Gamma(c+n)}{\Gamma(c+n-a)} U(a, c+n, z), \\
& y_{n}^{(3 \pm)}(z)=(-1)^{n} \Gamma(c+n) U\left(c+n-a, c+n, z e^{ \pm \pi i}\right),
\end{aligned}
$$

The asymptotic analysis of this case follows from the first connection formula in (2.5),

$$
y_{n}^{(1)}(z)={ }_{1} F_{1}(a ; c+n ; z)=e^{z}{ }_{1} F_{1}(c+n-a ; c+n ;-z),
$$

and by using the results of the $(++)$ recursion. It follows that

$$
\frac{y_{n+1}^{(1)}(z)}{y_{n}^{(1)}(z)} \sim 1, \quad n \rightarrow \infty,
$$

and the solution $y_{n}^{(1)}(z)$ is minimal in $|\mathrm{ph} z|<\pi$, in accordance with (7.11), whereas $y_{n}^{(2)}(z)$ and $y_{n}^{(3 \pm)}(z)$ are dominant.

7.3. The $(0-)$ recursion. The coefficients of this recursion can be obtained from (7.9),

$$
a_{n}=\frac{z(c-n-a)}{(c-n)(c-n-1)}, \quad b_{n}=\frac{1-c+n-z}{c-n-1} .
$$

Therefore,

$$
a_{n} \sim-\frac{z}{n}, \quad b_{n}=-1,
$$

and Perron's theorem establishes the following behavior of the solutions

$$
\frac{f_{n+1}(z)}{f_{n}(z)} \sim-\frac{z}{n}, \quad \frac{g_{n+1}(z)}{g_{n}(z)} \sim 1,
$$

the solution $f_{n}(z)$ being minimal.

Three solutions of this recursion are

$$
\begin{aligned}
& y_{n}^{(1)}(z)={ }_{1} F_{1}(a ; c-n ; z), \\
& y_{n}^{(2)}(z)=\frac{\Gamma(a+1-c+n)}{\Gamma(n+1-c)} U(a, c-n, z), \\
& y_{n}^{(3)}(z)=\frac{(-1)^{n} \Gamma(a+1-c+n)}{\Gamma(1-c+n) \Gamma(-c+n)} z^{n}{ }_{1} F_{1}(a+1-c+n, 2-c+n, z) .
\end{aligned}
$$


The reason for using the first connection formula in (2.4) instead of the second one is that we can now use again the results from the $(++)$ recursion to analyse the solution $y_{n}^{(3)}(z)$. It follows directly that

$$
\frac{y_{n+1}^{(3)}(z)}{y_{n}^{(3)}(z)} \sim-\frac{z}{n}, \quad n \rightarrow \infty,
$$

and the solution $y_{n}^{(3)}(z)$ is minimal in $|\mathrm{ph} z|<\pi$, in accordance with (7.17), whereas $y_{n}^{(1)}(z)$ and $y_{n}^{(2)}(z)$ are dominant.

7.4. The $(--)$ recursion. In this case, the recursion for the Kummer ${ }_{1} F_{1}$ function [1, Eq. 13.4.14] leads to an inconclusive result when applying Perron's theorem. However, if we use the recursion corresponding to the $U$ function [1, Eq. 13.4.27], then the coefficients are

$$
a_{n}=-z(a-n), \quad b_{n}=c-n-1-z .
$$

Therefore,

$$
a_{n} \sim-z n, \quad b_{n}=-n,
$$

and Perron's theorem establishes the following behavior of the solutions

$$
\frac{f_{n+1}(z)}{f_{n}(z)} \sim z, \quad \frac{g_{n+1}(z)}{g_{n}(z)} \sim n,
$$

the solution $f_{n}(z)$ being minimal.

Three solutions of this recursion are

$$
\begin{aligned}
& y_{n}^{(1)}(z)=\Gamma(1-c+n){ }_{1} F_{1}(a-n ; c-n ; z), \\
& y_{n}^{(2)}(z)=U(a-n, c-n, z), \\
& y_{n}^{(3)}(z)=\frac{\Gamma(-a+n+1)}{\Gamma(2-c+n)} z^{n}{ }_{1} F_{1}(a+1-c, 2-c+n, z) .
\end{aligned}
$$

Now we can use the results from the $(0+)$ recursion to analyse the solution $y_{n}^{(3)}(z)$, and it follows that

$$
\frac{y_{n+1}^{(3)}(z)}{y_{n}^{(3)}(z)} \sim z, \quad n \rightarrow \infty .
$$

Hence the solution $y_{n}^{(3)}(z)$ is minimal in $|\mathrm{ph} z|<\pi$, in accordance with (7.22), whereas $y_{n}^{(1)}(z)$ and $y_{n}^{(2)}(z)$ are dominant.

\section{Concluding Remarks}

Minimal and dominant solutions of confluent hypergeometric three-term recurrence relations have been presented. The cases studied correspond to the functions ${ }_{1} F_{1}\left(a+\epsilon_{1} n, c+\epsilon_{2} n, z\right)$ and $U\left(a+\epsilon_{1} n, c+\epsilon_{2} n, z\right)$, where $n$ is an integer parameter and $\epsilon_{i}=0, \pm 1$ (not both equal to 0 ). This produces eight different recursions, whose solutions can be analysed by applying Perron's theorem together with asymptotic estimates obtained for Whittaker functions with large values of the parameters. Some cases are related to each other, but for the sake of completeness we have considered each case separately. However, by using connection formulas, the analysis 
for related cases could be simplified. The results hold for complex values of $a$ and $c$ and complex values of $z$ in $|\mathrm{ph} z|<\pi$.

\section{Appendix: Asymptotic estimates of Whittaker functions}

We summarize the asymptotic estimates of the Whittaker functions as given in 2. From these estimates the asymptotic estimates of the Kummer functions follow by using the relations in (2.9).

Let

$$
\xi=\frac{z}{\kappa}, \quad \alpha=\frac{2 \mu}{\kappa}, \quad \xi_{1}=2-\sqrt{4-\alpha^{2}}, \quad \xi_{2}=2+\sqrt{4-\alpha^{2}} .
$$

Then the differential equation (2.6) can be written in the form

$$
\frac{d^{2}}{d \xi^{2}} w(\xi)=\left[\kappa^{2} \frac{\left(\xi-\xi_{1}\right)\left(\xi-\xi_{2}\right)}{4 \xi^{2}}-\frac{1}{4 \xi^{2}}\right] w(\xi) .
$$

We consider large positive values of $\kappa$, with $\mu / \kappa \in[-1+\delta, 1-\delta], 0<\delta<1, \delta$ being fixed. The differential equation has turning points at $\xi_{1}$ and $\xi_{2}$ when $\kappa$ is large. For $\xi$ in complex domains containing $\xi_{1}$ it is possible to derive asymptotic expansions in terms of Bessel functions, and in complex domains containing $\xi_{2}$ expansions in terms of Airy functions; see [2].

We give the relevant details on the Bessel case, because the Kummer recursions in this paper are considered for fixed $z$; hence, if $\kappa \rightarrow \infty$, the variable $\xi_{1}$ is small, and we need approximations that are valid for $\xi$ in domains near the origin 1

We have the following asymptotic representations (see [2, $\$ 6]$ ):

$$
\begin{aligned}
& M_{\kappa, \mu}(z)=F_{\kappa, \mu}(z) e^{\mu} \Gamma(2 \mu+1) \Psi_{\kappa, \mu} \\
& \quad \times\left[J_{2 \mu}(\kappa \sqrt{\zeta}) A_{\kappa, \mu}(z)+\frac{\sqrt{\zeta}}{\kappa} J_{2 \mu}^{\prime}(\kappa \sqrt{\zeta}) B_{\kappa, \mu}(z)\right] \\
& W_{\kappa, \mu}(z)=G_{\kappa, \mu}(z) e^{\kappa} \kappa^{-\kappa} \Psi_{\kappa, \mu} \Gamma\left(\kappa+\mu+\frac{1}{2}\right) \Gamma\left(\kappa-\mu+\frac{1}{2}\right) \\
& \quad \times\left[\left\{J_{2 \mu}(\kappa \sqrt{\zeta}) \sin (\kappa-\mu) \pi-Y_{2 \mu}(\kappa \sqrt{\zeta}) \cos (\kappa-\mu) \pi\right\} A_{\kappa, \mu}(z)\right. \\
& \left.\quad+\frac{\sqrt{\zeta}}{\kappa}\left\{J_{2 \mu}^{\prime}(\kappa \sqrt{\zeta}) \sin (\kappa-\mu) \pi-Y_{2 \mu}^{\prime}(\kappa \sqrt{\zeta}) \cos (\kappa-\mu) \pi\right\} B_{\kappa, \mu}(z)\right], \\
& W_{-\kappa, \mu}\left(z e^{-\pi i}\right)=H_{\kappa, \mu}(z) e^{\kappa} \kappa^{-\kappa} \Psi_{\kappa, \mu} \\
& \quad \times\left[H_{2 \mu}^{(1)}(\kappa \sqrt{\zeta}) A_{\kappa, \mu}(z)+\frac{\sqrt{\zeta}}{\kappa} H_{2 \mu}^{(1) \prime}(\kappa \sqrt{\zeta}) B_{\kappa, \mu}(z)\right] \\
& W_{-\kappa, \mu}\left(z e^{\pi i}\right)=I_{\kappa, \mu}(z) e^{\kappa} \kappa^{-\kappa} \Psi_{\kappa, \mu} \\
& \quad \times\left[H_{2 \mu}^{(2)}(\kappa \sqrt{\zeta}) A_{\kappa, \mu}(z)+\frac{\sqrt{\zeta}}{\kappa} H_{2 \mu}^{(2) \prime}(\kappa \sqrt{\zeta}) B_{\kappa, \mu}(z)\right]
\end{aligned}
$$

Here

$$
\Psi_{\kappa, \mu}=\frac{(\kappa-\mu)^{(\kappa-\mu) / 2}}{(\kappa+\mu)^{(\kappa+\mu) / 2}}
$$

and $F_{\kappa, \mu}(z), G_{\kappa, \mu}(z), H_{\kappa, \mu}(z)$, and $I_{\kappa, \mu}(z)$ are quantities that are not relevant in the present discussion, because

$$
\limsup _{\kappa \rightarrow \infty}\left|F_{\kappa, \mu}(z)\right|^{1 / \kappa}=1, \quad \limsup _{\kappa \rightarrow \infty}\left|\frac{F_{\kappa+k, \mu+m}(z)}{F_{\kappa, \mu}(z)}\right|=1,
$$

\footnotetext{
${ }^{1}$ The factor $e^{\kappa}$ on the right-hand sides of (9.4)-(9.6) was included after private communications with Mark Dunster.
} 
where $k$ and $m$ are fixed numbers. Similarly for $G_{\kappa, \mu}(z), H_{\kappa, \mu}(z)$, and $I_{\kappa, \mu}(z)$. The functions $A_{\kappa, \mu}(z)$ and $B_{\kappa, \mu}(z)$ have the asymptotic expansions

$$
A_{\kappa, \mu}(z) \sim \sum_{s=0}^{\infty} \frac{A_{s}(\zeta)}{\kappa^{2 s}}, \quad B_{\kappa, \mu}(z) \sim \sum_{s=0}^{\infty} \frac{B_{s}(\zeta)}{\kappa^{2 s}}, \quad \kappa \rightarrow \infty,
$$

uniformly with respect to $z$ in a bounded complex domain that contains the origin, with $-\pi<\operatorname{ph} z \leq \pi$. Details on the coefficients in these expansions are not needed in our analysis because $A_{\kappa, \mu}(z)$ and $B_{\kappa, \mu}(z)$ have the same limsup behavior as in (9.8).

The quantity $\zeta$ is defined in [2, Eq. (6.2)] 2 In the present discussion, because $|\xi|$ is small (see (9.1)), we use ([2, Eq. (3.9)])

$$
\zeta=c(\alpha) \xi+\mathcal{O}\left(\xi^{2}\right), \quad \xi \rightarrow 0,
$$

where

$$
c(\alpha)=\frac{4}{e \kappa} \sqrt{\kappa^{2}-\mu^{2}}\left(\frac{\kappa+\mu}{\kappa-\mu}\right)^{\kappa /(2 \mu)},
$$

which follows from operating in [2, Eq. (3.10)]).

Combining these two relations, we decide to use the following argument for the Bessel and Hankel functions that are needed:

$$
\kappa \sqrt{\zeta} \sim \kappa \sqrt{c(\alpha) \xi}=\sqrt{c(\alpha) \kappa z} .
$$

\section{ACKNOWLEDGEMENTS}

The authors acknowledge financial support from Ministerio de Educación y Ciencia, project MTM2006-09050. The authors thank Mark Dunster for helpful discussions on the asymptotic formulas for the Whittaker functions.

\section{REFERENCES}

[1] M. Abramowitz and I. A. Stegun, Handbook of mathematical functions with formulas, graphs, and mathematical tables, National Bureau of Standards Applied Mathematics Series, vol. 55, U.S. Government Printing Office, Washington, 1964. MR29:4914

[2] T. M. Dunster, Uniform asymptotic expansions for Whittaker's confluent hypergeometric functions, SIAM J. Math. Anal. 20 (1989), no. 3, 744-760. MR.990876 (90e:33012)

[3] W. Gautschi, Computational aspects of three-term recurrence relations, SIAM Review 9 (1967), no. 1, 26-82. MR0213062 (35:3927)

[4] A. Gil, J. Segura, and N. M. Temme, Numerical methods for special functions, SIAM, Philadelphia, PA, 2007.

[5] A. Gil, J. Segura, and N. M. Temme, The ABC of hyper recursions, J. Comp. Appl. Math 190 (2006), no. 1, 270-286. MR2209508(2006m:33003)

[6] _ Numerically satisfactory solutions of hypergeometric recursions, Math. Comp 76 (2007), no. 259, 1449-1468. MR2299782

[7] L. J. Slater, Confluent hypergeometric functions, Cambridge University Press, New York, 1960. MR0107026 (21:5753)

[8] N. M. Temme, Special functions, A Wiley-Interscience Publication, John Wiley \& Sons Inc., New York, 1996, An introduction to the classical functions of mathematical physics. MR.97e:33002

[9] N. M. Temme, Uniform asymptotic expansions of Laplace integrals, Analysis 3 (1983), 221249. MR.756117 (85j:41059)

\footnotetext{
${ }^{2}$ On the left-hand side of that equation $\kappa$ should be replaced by $\kappa^{2}$ (3 times).
} 
DamtP, Centre for Mathematical Sciences, University of Cambridge, Wilberforce RoAd, CB3 0WA, United Kingdom

E-mail address: ad495@cam.ac.uk

Departamento de Matemáticas, Estadística y Computación, Universidad de Cantabria, 39005 Santander, Spain

E-mail address: javier.segura@unican.es

CWI, P.O. Box 94079, 1090 GB Amsterdam, The Netherlands

E-mail address: nicot@cwi.nl 